\title{
Easier lateral orbitotomy approach for excision of lacrimal gland tumour
}

\author{
RL Sharma ${ }^{1, *}$, Janak Raj $^{2}$, Shagun Korla ${ }^{3}$ \\ ${ }^{1}$ Professor, ${ }^{2}$ Assistant Professor, ${ }^{3}$ Resident, ${ }^{1,3}$ Dept. of Ophthalmology, ${ }^{2}$ Dept. of Neurosurgery, Indira Gandhi Medical College, \\ Shimla, Himachal Pradesh, India \\ *Corresponding Author: \\ Email: rls_10@rediffmail.com
}

\begin{abstract}
Tumours of the lacrymal gland are relatively rare. Pleomorphic adenomas present with painless, unilateral progressive proptosis with downward and inward displacement of the globe. Surgical excision through the lateral orbitotomy is the commonest approach. It can be further simplified by removal of lateral wall with chisel instead of saw and replacing it back without any sutures or screws.
\end{abstract}

Keywords: Lacrymal gland tumour, Lateral orbitotomy, Pleomorphic adenoma.

\section{Introduction}

The most common benign neoplasm of the lacrimal gland is the pleomorphic adenoma. The term "benign mixed tumor" came from an earlier hypothesis, which remains popular, that these tumors derive from a mixture of epithelial and mesodermal elements. In fact, these tumors are epithelial in origin. Ductal epithelium develops into the epithelial component, and cells in the myoepithelium develop into cells in the stroma. The World Health Organization proposed the name pleomorphic adenoma, which more accurately describes the nature of the neoplasm. Pleomorphic adenoma accounts for approximately $12-25 \%$ of all lacrimal glands tumours.

Morphologically tumour is firm in consistency with a lobulated surface. The orbital lobe of the gland is most commonly involved. The tumour tends to extend backwards \& may cause proptosis, ophthalmoplegia, choroidal folds. Less commonly the tumour arises from the palpebral lobe. It tends to grow anteriorly, does not displace the globe \& produces early visible swelling of the upper eyelid. Although this tumour is histologically benign, incomplete excision is likely to result in recurrences leading to the increased orbital dysfunction $\&$ even malignant transformation. ${ }^{2}$ Therefore, when pleomorphic adenoma is suspected, a lateral orbitotomy should be done.

\section{Case}

A 37 year old female presented with history of painless, progressive protrusion of left eye since 1 year. It was associated with drooping of upper lid since 4 months. There was no history of blurring of vision, double vision and foreign body sensation in the eye. No history of fever, nausea, vomiting and weight loss. General physical and systemic examination was within normal limits.

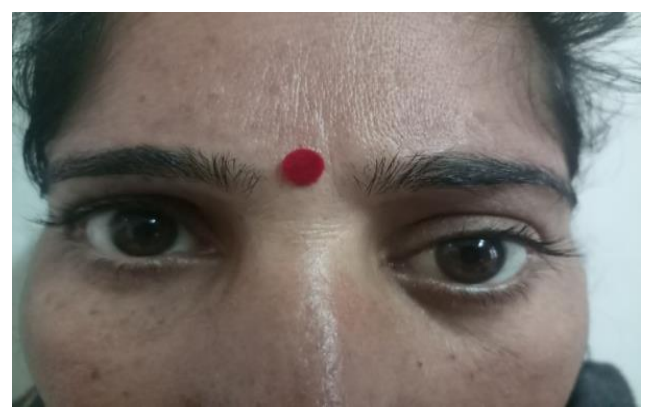

Fig. 1: Inferomedial displacement of the eyeball

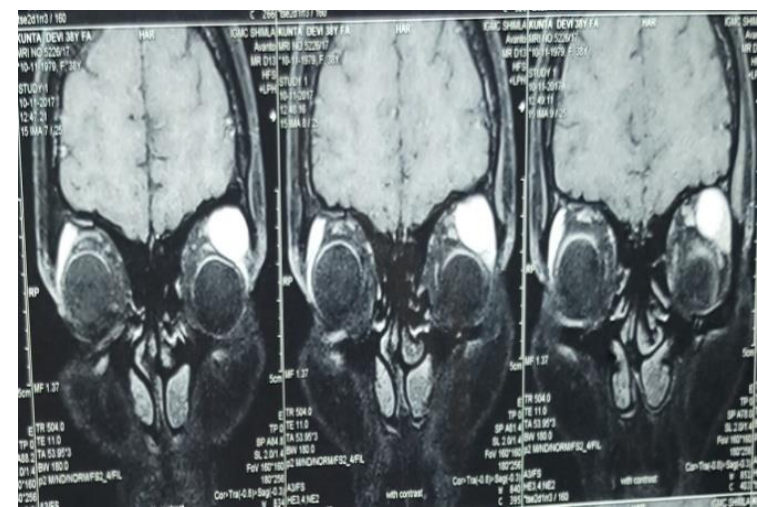

Fig. 2: MRI showing contrast enhanced lacrimal gland tuomor

On ocular examination visual acuity in both the eyes was $6 / 6$. Ocular movements were full and free in both the eyes. There was hypotropia of 10 degree in left eye (Fig. 1). Fundus examination was normal in both the eyes. Blood profile including renal function, liver function, thyroid function tests were within normal limits. MRI orbit showed altered signal intensity mass of size 19x16x12 mm on superolateral aspect of left orbit, which was compressing the left globe leading to its inferomedial displacement. Optic nerves extra-ocular muscles and fat were normal in bulk and no extension to the brain or bone was seen (Fig. 2). Radiologically the possibility of mixed tumour of lacrimal gland was kept.

Surgical excision of the tumour was planned through left lateral orbitotomy. The surgical technique 
involved a curvilinear incision on the supero-lateral orbital rim extending through the junction of lateral and inferior orbital margin. Dissection was carried out through the various subcutaneous layers including the orbicularis muscle to the level of the periosteum, which was reflected along the frontal process of zygomatic bone (Fig. 3).Then about 40mm segment of the frontal process of the zygomatic bone was removed using chisel and hammer to make horizontal cuts in the superior and inferior bony margins.
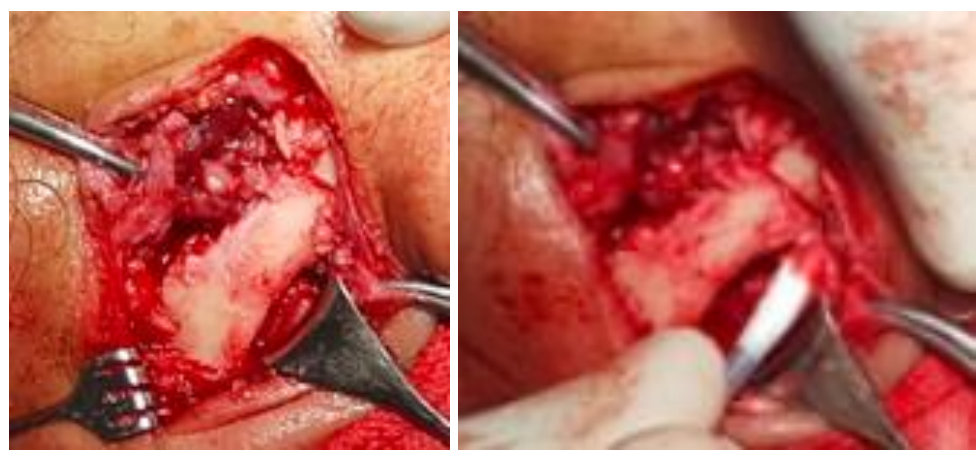

Fig. 3: Frontal process of zygomatic before $\&$ after

\section{Fig. 4: Lacrimal gland tumour}

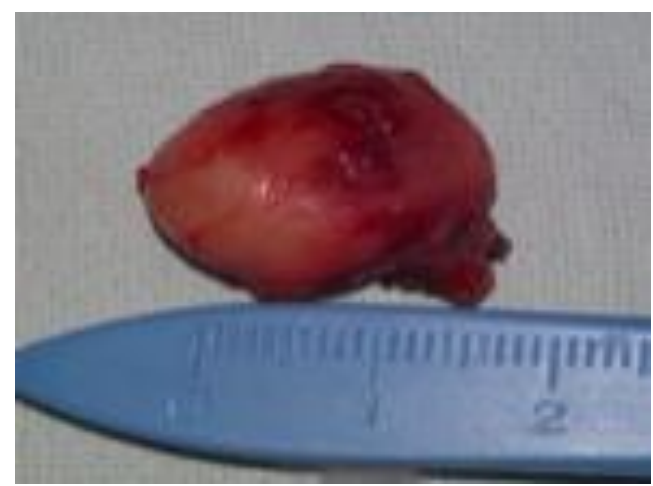

The mass was removed in to along with its psuedocapsule (Fig. 4). The frontal process of zygomatic bone was replaced back with good fixation without the use of any sutures or screws. There was no bony tissue loss as is seen with use of saw. The spared periosteum was sutured with vicryl 3-0 to secure the bony segment in place, overlying muscles and skin was sutured.
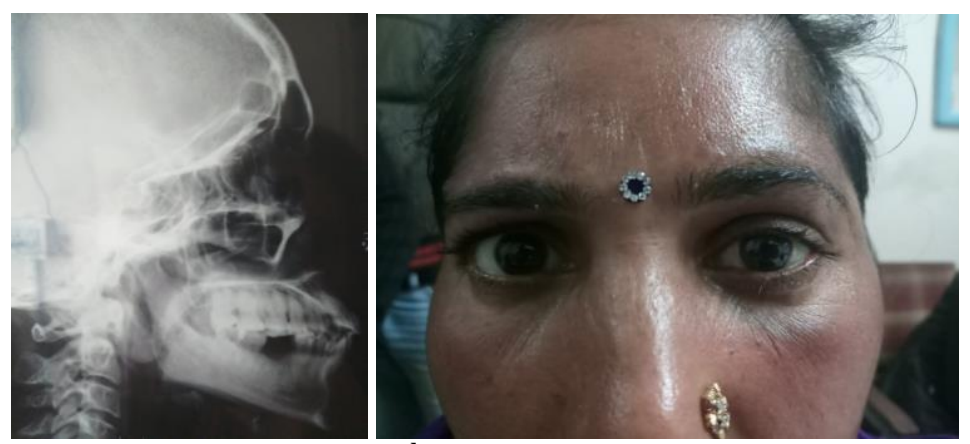

Fig. 5: Postoperative X-Ray and photograph at $3^{\text {rd }}$ month

Histopathology of specimen showed pleomorphic adenoma of lacrimal gland. Postoperatively there was lid edema, which subsided in 5 days. Vision, ocular movements and fundus was normal at 3 months follow up (Fig. 5).

\section{Discussion}

Tumours of the lacrimal gland are relatively rare. The incidence in various studies has been found to be between 5 to $7 \%$. Pleomorphic adenoma accounts for approximately $12-25 \%$ of all lacrimal glands tumours. ${ }^{1,2}$ It has been said that about $50 \%$ of lacrimal masses are epithelial and $50 \%$ are nonepithelial. ${ }^{2}$ Pleomorphic adenoma is most common benign tumour of the lacrimal 
glands. Pleomorphic adenomas usually occur in the fourth and fifth decade of life, and incidence is equal for both genders. There may be cystic areas within the mass \& long standing lesions may erode adjacent bone. Although pleomorphic adenomas commonly involve the orbital lobe of the lacrimal gland, they can involve the palpabral lobe in about $10 \%$ of cases. Malignant transformation can occur in 10-20\% of pleomorphic adenomas of the lacrimal gland. ${ }^{3,4}$ Seventy-five percent of pleomorphic adenomas transform into pleomorphic adenocarcinoma, while the rest transform into cystic carcinoma. $^{4}$

Pleomorphic adenomas commonly present with symptoms of painless, unilateral progressive proptosis with downward and inward displacement of the globe. Other presenting symptoms or signs include diplopia or an ocular motility disturbance, a change in refractive error, orbital discomfort, lacrimation, ptosis, and choroidal folds. A palpable mass in the superotemporal orbital quadrant is present in most patients and is nontender. The palpebral lobe tumors are freely movable\& present within a shorter duration. They do not produce proptosis or bony changes.

CT images show round to oval, well-defined lesions that are smooth in outline, displacing and deforming the globe. The contrast enhancement is moderate to marked. Some long-standing large tumors show lobulations and radiolucent areas of cystic degeneration. In the bone window, the lacrimal fossa is prominent owing to pressure erosion. This condition can progress to a defect in the orbital roof with contact of tumor and duramater. On MRI the tumor is of low signal intensity on the T1weighted image and of high signal intensity on the T2weighted image, frequently of heterogeneous distribution with intense contrast enhancement. ${ }^{5}$ On USG studies, pleomorphic adenomas are medium to highly reflective, with a regular structure \& moderate sound attenuation. ${ }^{6}$

The best management is complete excision of the tumor within its pseudocapsule via a lateral orbitotomy. The lateral orbitotomy is classically done by drilling hole in frontal process and electric saw which usually cause some bony tissue loss requiring fixation titanium plating with multiple screw. But in this case it was done using chisel and hammer to make horizontal cuts in the superior and inferior bony margins removing about $40 \mathrm{~mm}$ segment of the frontal process of the zygomatic bone. The frontal process of zygomatic bone can be replaced back with good fixation without the use of any sutures or screws, as was done in this case. The bony fragment stays well in position provided it is fixed with spared periosteum. There was no bony tissue loss as is seen with use of saw. The spared periosteum was sutured with vicryl 3-0 to secure the bony segment in place, overlying muscles and skin was sutured.

To minimize any tumor seeding from microscopic extensions through the pseudocapsule, an adequate margin of the surrounding lacrimal gland and the adjacent periorbita should be removed in the extirpation. ${ }^{7,8}$ When biopsy is done before excision, the $5 \mathrm{yr}$ recurrence rate is estimated to be $32 \%$ \& many of these recurrences undergo malignant transformation. ${ }^{9}$ Font \& Gamel have emphasized the high recurrence rate following incomplete excision or incisional biopsy. The $5 \mathrm{yr}$ recurrence rate was $3 \%$ for completely excised lesions \& 32\% for incompletely excised tumours. Recurrent pleomorphic adenoma can undergo malignant change. Font \& Gamel reported that about $10 \%$ of adenomas undergo malignant change by $20 \mathrm{yrs}$ after $1 \mathrm{st}$ treatment $\& 20 \%$ by 30 yrs. $^{9}$

The lateral approach was 1 st proposed by Kronlein in 1889 , and it was later modified by Berke. ${ }^{10}$ Wirtschafter et al. described preservation of the lateral rim in lateral orbitotomy. ${ }^{11}$ Some advocate additional removal of the palpebral lobe of the lacrimal gland with excretory ductules to reduce the recurrence rate. ${ }^{12}$ However, preservation of the palpebral lobe greatly reduces the incidence of postoperative dry eye \& the need for topical lubricants. Another approach called transcranial orbital technique can be used to remove orbital lesions. ${ }^{13}$ Complications like decrease in visual acuity, diplopia, gaze paresis and enophthalmos could develop more after transcranial approaches. ${ }^{14}$ Nevertheless, lateral orbitotomy is still an appropriate, safe and less time-consuming procedure for lateral extraconal lesions of the orbit. ${ }^{15}$

We conclude that surgical excision through the lateral orbitotomy approach without use of any sutures or screws is an effective modality to approach orbital tumour like pleomorphic adenoma of the lacrimal gland.

\section{Conflicts of Interest: Nil}

\section{References}

1. Shield CL, Shield JA, Eagle RC, Rathwell JP. Clinicopathologic review of 142 cases of lacrimal gland lesions. Ophthalmology 1989;96:431-434.

2. Reese AB. Expanding lesions of the orbit. Bowan lecture. Trans Ophthalmol Soc UK 1971, 91:90- 98.

3. Perzin KH, Jakobiec FA, Livolsi VA, Desjardins L. Lacrimal gland malignant mixed tumors (carcinomas arising in benign mixed tumors). Cancer 1980;45:2593606.

4. Wright JE, Rose GE, Garner A. Primary malignant neoplasms of the lacrimal gland. Br J Ophthalmol 1992;76:401-7.

5. Hosten N, Bornfeld N. Imaging of the Globe and Orbit. A Guide to Differential Diagnosis. Stuttgart-New York: Thieme; 1998:110-122.

6. Byrne SF, Green RL. Ultrasound of the Eye \& Orbit. 2nd ed. St. Louis, Mo: Mosby;2002:324.

7. Henderson JW. Orbital tumors. 3rd ed. New York :Raven Press;1994:323-342.

8. Rose GE, Wright JE. Pleomorphic adenoma of the lacrimal gland. Br J Ophthalmol 1992;76:395-400. 
9. Font RL, Gamel JW. Epithelial tumors of the lacrimal gland: an analysis of 265 cases In: Jacobeic FA, ed. Ocular \& Adnexal tumors. Bimingham, AL: Aesculpius Publishing, 1978:787-805.

10. Berke RN: Modified Kronlein operation. AMA Arch Ophth 51:609-632, 1954

11. Wirtschafter JD, Chu AE. Lateral orbitotomy without removal of the lateral orbital rim. Arch Ophthalmol (1988) 106:1463-1468.

12. Rootman JA: Diseases of the orbit: A Multidisciplinary Approach. Philadelphia: JB Lippincott, 1988:384-405.

13. Margalit N, Ezer H, Fliss DM, Naftaliev E, Nosek E, Kesler A: Orbital tumors treated using transcranial approaches: surgical technique and neuroophthalmogical results in 41 patients. Neurosurg Focus 23:E1, 200713.
14. Kang JK, Lee IW, Jeun SS, Choi YK, Jung CK, Yang JH, Kim DS: Tumors of the orbit. Pitfalls of the surgical approach in 37 children with orbital tumor. Child Nerv Syst 13:536-541, 1997

15. Ram Lal Sharma, Roop Chand Thakur, Chandan Sud, Arti Sareen: Extended lateral orbitotomy, an effective procedure to excise large orbital hydatid cyst: A rare case. Annals of Tropical Medicine and Public Health | Jan Jun 2011 | Vol 4 |Issue 1;45-47. 